\title{
CARACTERIZAÇÃO DO MATERIAL PARTICULADO FINO E GROSSO E COMPOSIÇÃO DA FRAÇÃO INORGÂNICA SOLÚVEL EM ÁGUA EM SÃO JOSÉ DOS CAMPOS (SP)
}

\section{Patricia Alexandre de Souza e William Zamboni de Mello*}

Departamento de Geoquímica, Instituto de Química, Universidade Federal Fluminense, Outeiro de São João Batista, s/n, 24020-141 Niterói - RJ, Brasil

Rauda Lúcia Mariani\#

Centro de Previsão do Tempo e Estudos Climáticos, Instituto Nacional de Pesquisas Espaciais, CP 515, 12227-010 São José dos Campos - SP, Brasil

\section{Silvia Maria Sella}

Departamento de Química Analítica, Instituto de Química, Universidade Federal Fluminense, Outeiro de São João Batista, s/n, 24020-141 Niterói - RJ, Brasil

\begin{abstract}
CARACTERIZATION OF FINE AND COARSE PARTICULATE MATTER AND COMPOSITION OF THE WATER-SOLUBLE INORGANIC FRACTION IN SÃO JOSÉ DOS CAMPOS (SP). Air samples of fine $\left(\mathrm{PM}_{2,5}\right)$ and coarse $\left(\mathrm{PM}_{2,5-10}\right)$ particulate matter were collected in São José dos Campos from February 2004 to February 2005. Average $\mathrm{PM}_{10}$ mass concentrations was $31.2 \pm 14.0$ $\mu \mathrm{g} \mathrm{m}^{-3}$, half of which belonging to the $\mathrm{PM}_{2.5}$ fraction. Ammonium and $\mathrm{SO}_{4}{ }^{2-}$ were predominantly found in the fine fraction. Average $\left(\mathrm{NH}_{4}\right)_{2} \mathrm{SO}_{4}$ concentration was estimated to be about $2.9 \mu \mathrm{g} \mathrm{m}{ }^{-3}$. Chloride, $\mathrm{Na}^{+}$and $\mathrm{NO}_{3}{ }^{-}$were mostly associated with $\mathrm{PM}_{2,5-10^{*}}$. Chloride deficits with respect to sea-salt $\mathrm{Cl} / \mathrm{Na}$ ratio were found in both size fractions.
\end{abstract}

Keywords: airborne particulate matter; dichotomous; ammonium sulfate.

\section{INTRODUÇÃO}

O material particulado (MP) atmosférico é conhecido como uma mistura complexa de partículas líquidas e sólidas (aerossóis), de tamanho na faixa entre poucos nanômetros até aproximadamente 100 $\mu \mathrm{m}$, em suspensão no ar. ${ }^{1,2}$ Essa faixa de tamanho compreende desde núcleos constituídos por poucas moléculas agrupadas até gotículas de nuvens e partículas provenientes da litosfera, hidrosfera e biosfera. Essas partículas podem ser emitidas diretamente de suas fontes ou formadas por reações químicas na atmosfera constituindo, respectivamente, as assim chamadas partículas primárias e secundárias.

As medidas das concentrações de MP na atmosfera têm objetivos tanto científico quanto de regulamentação. Estudos de caráter científico buscam, em geral, relacionar composição química e faixa de tamanho, com vistas à identificação das fontes do MP, ao entendimento das transformações químicas na atmosfera e ao provimento de subsídios para uma melhor compreensão dos efeitos adversos do MP à saúde humana e para as ações de controle ambiental. Nas últimas décadas, a influência das atividades humanas no aumento das concentrações de MP na atmosfera vem recebendo maior atenção também devido a sua contribuição como forçante negativa no balanço energético global e nas mudanças das propriedades físicas das nuvens. ${ }^{3}$

O tamanho do MP está diretamente relacionado ao seu potencial em provocar efeitos adversos à saúde humana. As partículas inaláveis (PI), ou seja, aquelas que possuem diâmetro aerodinâmico $\left(\mathrm{D}_{\mathrm{a}}\right)$ menor que $10 \mu \mathrm{m}$, são aquelas capazes de penetrar no sistema respiratório humano podendo ser depositadas desde as vias nasais até as partes mais profundas dos pulmões e, portanto, representam um risco para o desenvolvimento e a exacerbação de doenças respiratórias e

*e-mail: zamboni@geoq.uff.br

\# Endereço permanente: Departamento de Geoquímica, Instituto de Química, Universidade Federal Fluminense, Outeiro de São João Batista, s/n, 24020141 Niterói - RJ. cardiovasculares. ${ }^{4}$ As PI (outra abreviatura $\mathrm{MP}_{10}$ ) são comumente subdivididas em partículas inaláveis grossas e finas (inhalable fine and coarse particles $),{ }^{5}$ sendo as primeiras aquelas de $\mathrm{D}_{\mathrm{a}}$ entre 2,5 e $10 \mu \mathrm{m}\left(\mathrm{MP}_{2,5-10}\right)$ e as últimas, as de $\mathrm{D}_{\mathrm{a}}$ menor que $2,5 \mu \mathrm{m}\left(\mathrm{MP}_{2,5}\right)$.

As partículas primárias tendem a constituir a fração grossa do MP e as secundárias a fração fina. As partículas grossas são geralmente produzidas mecanicamente pela fragmentação de partículas maiores e transferidas para a atmosfera pela ação dos ventos, como é o caso da poeira do solo, dos fragmentos de folhas, dos grãos de pólen e dos borrifos de água do mar (sea salt spray). ${ }^{1,2}$ As partículas finas são formadas na atmosfera a partir da condensação de vapores quentes, seguida de coagulação e aglomeração, e de reações entre gases (processos de conversão gás-partícula). ${ }^{1,2}$

No Brasil, dentre as principais atividades antrópicas poluidoras do ar atmosférico em regiões urbanas e industrializadas destacam-se a queima de combustíveis fósseis, os processos industriais, a incineração do lixo urbano, a mineração, a construção civil e as práticas agrícolas inadequadas associadas às atividades de queimadas. São vários os trabalhos brasileiros relacionados à composição química do MP atmosférico em áreas influenciadas pelas emissões veiculares, industriais e atividades relacionadas à produção de energia, ${ }^{6}$ entretanto, são poucos os estudos em áreas urbanas relacionados à composição química de substâncias inorgânicas solúveis em água associadas às PI coletadas por equipamentos que permitam seu fracionamento sob as formas de $\mathrm{MP}_{2,5}$ e MP ${ }_{2,5-10^{7}}{ }^{7-10}$ No que tange à influência da queima de biomassa, associada à colheita da cana-de-açúcar e ao desmatamento de áreas do cerrado e da floresta tropical amazônica, sobre a composição química do MP atmosférico, Allen et al. ${ }^{11}$ investigaram a composição iônica (inorgânica e orgânica) em amostras de MP fino $\left(D_{a}<3,5 \mu \mathrm{m}\right)$ e grosso $\left(D_{a}>3,5 \mu \mathrm{m}\right)$ coletadas em área rural nas proximidade de Araraquara (SP) e Yamasoe et al. ${ }^{12} \mathrm{em}$ amostras de MP fino $\left(D_{a}<2,0 \mu \mathrm{m}\right)$ e grosso $\left(D_{a}>2,0 \mu \mathrm{m}\right)$ coletadas, no período agosto-setembro, na Reserva Ecológica do Instituto Brasileiro de Geografia e Estatística, ao sul de Brasília, e em Jamari (RO). 
O presente estudo teve como objetivo quantificar e determinar a distribuição da fração fina e grossa das PI, coletadas através de um amostrador de $\mathrm{MP}_{10}$ dicotômico, bem como quantificar e determinar a distribuição dos principais constituintes inorgânicos solúveis em água $\left(\mathrm{Na}^{+}, \mathrm{K}^{+}, \mathrm{Ca}^{2+}, \mathrm{Mg}^{2+}, \mathrm{NH}_{4}^{+}, \mathrm{Cl}^{-}, \mathrm{NO}_{3}{ }^{-}\right.$e $\left.\mathrm{SO}_{4}{ }^{2-}\right)$ nessas frações, em São José dos Campos (SP).

\section{PARTE EXPERIMENTAL}

\section{Área de estudo}

O município de São José dos Campos (SJC) possui uma população em torno de 600 mil habitantes e está localizado na porção média da bacia do Rio Paraíba do Sul (Vale do Paraíba) a cerca de $90 \mathrm{~km}$ a nordeste da capital do estado de São Paulo (SP) (Figura 1S, material suplementar) e a uma distância mínima de aproximadamente 65 km do mar (direção norte-sul). O município é cortado pela Rodovia Presidente Dutra (BR-116), que liga a Região Metropolitana de São Paulo (RMSP) à Região Metropolitana do Rio de Janeiro (RMRJ).

A área total do município é de $1.102 \mathrm{~km}^{2}$, sendo $70 \%$ constituída por montanhas, serras e picos associados à formação da Serra do Mar e Mantiqueira e os 30\% restantes por um imenso planalto, onde se concentra toda a população urbana do município. ${ }^{13}$ A cidade de SJC encontra-se a uma altitude média de $600 \mathrm{~m}$ e o clima é tropical sub-quente úmido, caracterizado por um período seco durante o outono-inverno e um chuvoso correspondente à primavera-verão. Os ventos predominantes são os de norte-nordeste (NNE; 17\%) e nordeste (NE; 13\%), havendo também contribuições significativas oriundas das direções sul-sudoeste (SSW; 10\%) e sul-sudeste (SSE; $10 \%) .{ }^{14}$ A calmaria (velocidade do vento $<0,5 \mathrm{~m} \mathrm{~s}^{-1}$ ) prevalece por $25 \%$ do período. ${ }^{14} \mathrm{~A}$ precipitação apresenta variações devido à presença de um relevo diversificado que influi na circulação geral dos ventos, que regulam a distribuição das chuvas. ${ }^{15}$ As médias anuais variam de 1.300 a $2.000 \mathrm{~mm}$ na Serra da Mantiqueira e de 1.300 a $2.800 \mathrm{~mm}$ na Serra do Mar. ${ }^{13}$

O parque industrial de SJC é constituído por aproximadamente 900 empresas de diferentes segmentos, como automotiva, aeroespacial, de telecomunicações, química e petroquímica. ${ }^{16}$ Sua frota automotiva é constituída de aproximadamente 281 mil veículos. ${ }^{17} \mathrm{De}$ acordo com a Companhia de Tecnologia de Saneamento Ambiental (CETESB),${ }^{14}$ o município emite anualmente (ano base 2006) $14,7 \mathrm{Gg}$ de óxidos de nitrogênio $\left(\mathrm{NO}_{\mathrm{x}}=\mathrm{NO}+\mathrm{NO}_{2}\right), 9,6 \mathrm{Gg}$ de dióxido de enxofre $\left(\mathrm{SO}_{2}\right)$ e $2,1 \mathrm{Gg}$ de MP para a atmosfera, dos quais, respectivamente, 39,97 e $62 \%$ provêm de fontes fixas. $\left(1 \mathrm{G}=10^{9}\right)$

\section{Amostragem}

As amostras de $\mathrm{MP}$ fino $\left(\mathrm{MP}_{2,5}\right)$ e grosso $\left(\mathrm{MP}_{2,5-10}\right)$ foram coletadas de 24/2/2004 a 24/2/2005 no Instituto Nacional de Pesquisas

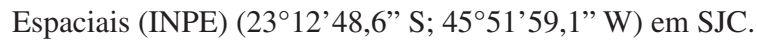

As coletas foram realizadas, durante $24 \mathrm{~h}$ em intervalos de 6 dias, através de um amostrador dicotômico $\mathrm{MP}_{10}$ Graseby Andersen modelo $241,{ }^{8}$ que separa as duas frações, conforme recomendações estabelecidas pela United States Environmental Protection Agency (US-EPA). ${ }^{18}$ Este equipamento consiste de um compartimento de controle e outro de amostragem. O compartimento de controle (41 x 56 x $28 \mathrm{~cm} ; 25 \mathrm{~kg}$ ) possui uma bomba de vácuo, os rotâmetros (para o controle da vazão total de entrada e da vazão de coleta do particulado grosso) e os reguladores de tempo de amostragem. Através do compartimento de controle são ajustados o horário de início da coleta e sua duração. O compartimento de amostragem (1,6 m de altura) engloba o sistema de entrada de ar (inlet), o impactador virtual (separador de partículas) e os suportes das membranas sobre as quais são retidas as amostras. De acordo com as especificações fornecidas pelo fabricante (Graseby Andersen Inc.), a estrutura da cabeça do coletor não permite interferências provocadas por ventos ou chuva, além disso, a mesma possui uma tela que impede a entrada de insetos. A vazão total de entrada de ar é de 16,7 $\mathrm{L} \mathrm{min}^{-1}$, da qual $90 \%$ correspondem à vazão de coleta do MP fino e $10 \%$ do MP grosso. As vazões dos dois rotâmetros foram calibradas no local de estudo por meio de fluxômetros Top-Trak modelo 822 (Sierrra Instruments, Inc.) específicos para as faixas $0-20 \mathrm{~L} \mathrm{~min}^{-1}$ (vazão total) e $0-2 \mathrm{~L} \mathrm{~min}^{-1}$ (vazão de coleta do particulado grosso).

As amostras foram coletadas em membranas de Teflon de 37 mm de diâmetro e 2,0 $\mu \mathrm{m}$ de porosidade (Sierra-Andersen Tef-Disc Teflon), fixadas por meio de anéis de polipropileno de $44,5 \mathrm{~mm}$ de diâmetro externo. Após a coleta as amostras foram armazenadas em freezer a $-19{ }^{\circ} \mathrm{C}$ para minimizar perdas por volatilização e estancar a ação de micro-organismos até que as extrações fossem efetuadas. Os filtros foram pesados, antes e após a coleta, numa balança analítica (Sartorius) com precisão de $10 \mu \mathrm{g}$. Antes das pesagens os filtros foram mantidos em dessecador por $24 \mathrm{~h}$. Wilson et al.${ }^{19}$ descreveram sucintamente sobre amostradores de PI e seu fracionamento em MP fino e grosso, bem como sobre a composição química dessas frações e as técnicas analíticas empregadas.

\section{Análises químicas}

Para a solubilização do filtro, o mesmo foi inserido num frasco de polipropileno de $100 \mathrm{~mL}$ de boca larga e nele adicionados $50 \mathrm{~mL}$ de água destilada (Fisatom, modelo 525) e deionizada (Permution) (resistividade $18 \mathrm{M} \Omega \mathrm{cm}$ ). Logo após esse procedimento, o frasco foi submetido ao ultrassom por $3 \mathrm{~h}$ e, em seguida, deixado em repouso por $12 \mathrm{~h} .{ }^{7}$ Os extratos obtidos foram transferidos para frascos de polipropileno e armazenados em freezer $\left(-19^{\circ} \mathrm{C}\right) . \mathrm{O} \mathrm{pH}$ e a condutividade foram medidos em alíquotas não filtradas em temperatura ambiente $\left(\sim 25^{\circ} \mathrm{C}\right)$ através de aparelhos WTW modelos pH330 e LF330. Para as medidas de $\mathrm{pH}$, utilizou-se eletrodo de vidro combinado calibrado com padrões 4,00 e $6,86 .{ }^{20} \mathrm{O}$ condutivímetro foi calibrado com soluções de 0,1 e $0,5 \mathrm{mmol} \mathrm{L}^{-1}$ de $\mathrm{KCl}$, cujas condutividades correspondem, respectivamente, a 14,9 e $73,9 \mu \mathrm{S} \mathrm{cm}^{-1}$ a $25^{\circ} \mathrm{C}$, os padrões mais baixos de calibração, recomendados pelo Standard Methods for the Examination of Water and Wastewater, para medidas de condutividade em amostras de água naturais e poluídas. ${ }^{20}$

$\mathrm{O}$ íon $\mathrm{NH}_{4}^{+}$foi determinado pelo método do indofenol, com as leituras das absorvâncias realizadas num espectrofotômetro Hitachi

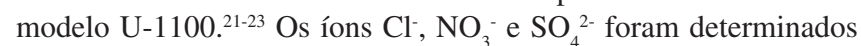
por cromatografia de íons, com detector condutimétrico, sem sistema de supressão iônica (Shimadzu, modelo LC-10AD). ${ }^{22,23}$ Nesse caso, as amostras foram filtradas, no momento da injeção, por meio de unidades filtrantes de acetato de celulose acopladas diretamente à seringa. Num equipamento Varian modelo Espectra AA-300 foram determinados os íons $\mathrm{Na}^{+}$e $\mathrm{K}^{+}$por espectrometria de emissão atômica e os íons $\mathrm{Mg}^{2+}$ e $\mathrm{Ca}^{2+}$ por espectrometria de absorção atômica.

Os cálculos dos limites de detecção dos íons analisados foram realizados levando-se em consideração também os brancos de filtros, ${ }^{24}$ cujas concentrações (em $\mu \mathrm{mol} \mathrm{L}{ }^{-1}$ ) resultantes de extrações de filtros (filtro em $50 \mathrm{~mL}$ de água) sem amostras foram: $\mathrm{NH}_{4}^{+}(0,42), \mathrm{Na}^{+}(3,66), \mathrm{K}^{+}$ $(2,33), \mathrm{Ca}^{2+}(1,96), \mathrm{Mg}^{2+}(0,15), \mathrm{Cl}^{-}(5,00), \mathrm{SO}_{4}^{2-}(1,43)$ e $\mathrm{NO}_{3}^{-}(0,50)$. Esses valores representam a média aritmética dos resultados obtidos das extrações de 7 filtros sem amostras. Com base num volume teórico de ar equivalente a $24 \mathrm{~m}^{3}\left(\mathrm{~T}=25^{\circ} \mathrm{C}\right.$ e $\left.\mathrm{P}=1 \mathrm{~atm}\right)$, os limites de quantificação $\left(e m \mu \mathrm{g} \mathrm{m}^{-3}\right)$ foram: $\mathrm{NH}_{4}^{+}(0,02), \mathrm{Na}^{+}(0,17), \mathrm{K}^{+}(0,11), \mathrm{Ca}^{2+}$ $(0,09), \mathrm{Mg}^{2+}(0,01), \mathrm{Cl}^{-}(0,24), \mathrm{SO}_{4}^{2-}(0,06)$ e $\mathrm{NO}_{3}^{-}(0,02)$.

A qualidade das análises para os íons $\mathrm{Cl}^{-}, \mathrm{SO}_{4}^{2-}, \mathrm{Na}^{+}, \mathrm{K}^{+}, \mathrm{Ca}^{2+}$ e $\mathrm{Mg}^{2+}$ foi verificada por meio de diluições de água do mar padrão 
(IAPSO Standard Seawater; salinidade = 34,996; Ocean Scientific International Ltd., UK) em água destilada e deionizada. Os resultados obtidos deste teste apresentaram variações, em relação às concentrações esperadas, na faixa de $\pm 10 \%$. A precisão das análises químicas, expressa como coeficiente de variação, esteve no intervalo de $\pm 5 \%$.

\section{RESULTADOS E DISCUSSÃO}

\section{Concentrações de partículas inaláveis, finas e grossas}

Os resultados das concentrações do PI $\left(\mathrm{MP}_{10}\right)$, MP fino $\left(\mathrm{MP}_{2,5}\right)$ e MP grosso $\left(\mathrm{MP}_{2,5-10}\right)$, obtidas em SJC no período de fevereiro de 2004 a fevereiro de 2005 , encontram-se compilados na Tabela 1. Esses resultados compreendem coletas efetuadas uma vez a cada 6 dias, que representariam quase $17 \%$ do total de dias do ano. Entretanto, efetivamente obteve-se pouco mais de $14 \%$ do total de dias de um ano. Embora as coletas tenham ocorrido em intervalos de 6 dias, doravante esses valores serão tratados como médias anuais, tal qual foi efetuado pela CETESB $^{25}$ na RMSP, utilizando um amostrador $\mathrm{MP}_{10}$ dicotômico, cujas coletas foram efetuadas com intervalos de 6 dias. As médias aritméticas ( \pm desvio padrão) das concentrações de $\mathrm{MP}_{10}, \mathrm{MP}_{25}$ e $\mathrm{MP}_{25-10}$ nesse período foram, respectivamente, $31,2 \pm 14,0,15,7 \pm 7,9$ e $14,8 \pm 8,4 \mu \mathrm{g} \mathrm{m}^{-3}$. A concentração média anual de $\mathrm{MP}_{10}$ em SJC foi inferior à média anual padrão (primário e secundário) estabelecida pelo Conselho Nacional do Meio Ambiente (CONAMA), ${ }^{26}$ que é de $50 \mu \mathrm{g} \mathrm{m}{ }^{-3}$, mas foi superior ao limite proposto pela Organização Mundial de Saúde (OMS), ${ }^{4}$ que é de $20 \mu \mathrm{g} \mathrm{m}^{-3}$.

Tabela 1. Concentrações médias anuais ( $\left(\mathrm{em} \mu \mathrm{g} \mathrm{m} \mathrm{m}^{-3} ; \mathrm{n}=52\right)$ de $\mathrm{MP}$ fino $\left(\mathrm{MP}_{2.5}\right)$ e grosso $\left(\mathrm{MP}_{2,5-10}\right)$ em São José dos Campos (SP) no período de fevereiro de 2004 a fevereiro de 2005

\begin{tabular}{lccccc}
\hline & $\mathrm{MA} \pm \mathrm{DP}$ & $\mathrm{CV} \%$ & Mediana & Mínimo & Máximo \\
\hline $\mathrm{MP}_{2,5}$ & $15,7 \pm 7,9$ & 52 & 14,8 & 1,1 & 40,7 \\
$\mathrm{MP}_{2,5-10}$ & $14,8 \pm 8,4$ & 56 & 13,9 & 1,1 & 38,4 \\
$\mathrm{MP}_{10}$ & $31,2 \pm 14,0$ & 45 & 31,8 & 3,3 & 71,9 \\
$\mathrm{MP}_{2,5} / \mathrm{MP}_{10}$ & $0,52 \pm 0,16$ & 31 & 0,49 & 0,17 & 0,95 \\
\hline
\end{tabular}

MA: Média aritmética; DP: Desvio padrão; CV: Coeficiente de variação.

Em se tratando de concentrações médias de $24 \mathrm{~h}$ para $\mathrm{MP}_{10}$, não houve valor que tenha superado o padrão (primário e secundário) estabelecido pelo CONAMA, ${ }^{26}$ que é de $150 \mu \mathrm{g} \mathrm{m}^{-3}$ (valor que não deve ser excedido mais de uma vez por ano). Em relação ao limite proposto pela $\mathrm{OMS}^{4}$ como média de $24 \mathrm{~h}$, que é de $50 \mu \mathrm{g} \mathrm{m}^{-3}$ (valor que não deve ser excedido mais de três vezes por ano), foram observados dois valores superiores a este limite $\left(71,9\right.$ e $\left.64,5 \mu \mathrm{g} \mathrm{m}^{-3}\right)$, ocorridos em setembro e outubro de 2004.

A concentração média anual do $\mathrm{MP}_{2,5}$ foi $15,7 \mu \mathrm{g} \mathrm{m}{ }^{-3}$. O CONA$\mathrm{MA}^{26}$ não estabelece padrão de qualidade para esta faixa de tamanho de partículas em suspensão no ar. Entretanto, a média anual encontrada superou o padrão proposto pela $\mathrm{OMS}^{4}$ para $\mathrm{MP}_{2,5}$, que é de $10 \mu \mathrm{g}$ $\mathrm{m}^{-3}$. No que tange às concentrações médias de $24 \mathrm{~h}$ para $\mathrm{MP}_{2,5}$, seis valores (que variaram de 26,0 a 40,7 $\mathrm{g} \mathrm{g} \mathrm{m}^{-3}$ ) ultrapassaram o limite proposto pela OMS, ${ }^{4}$ que é de $25 \mu \mathrm{g} \mathrm{m}{ }^{-3}$. Destes seis valores, cinco ocorreram no período de maio a outubro de 2004 e um em fevereiro. Novamente, ressalta-se que esta amostragem compreendeu apenas $14 \%$ do total de dias de um ano.

A média da razão $\mathrm{MP}_{2,5} / \mathrm{MP}_{10}$ é $0,52 \pm 0,17$ (Tabela 1 ), o que significa que, em média, metade da massa total do $\mathrm{MP}_{10}$ é atribuída ao MP fino. De acordo com a OMS, ${ }^{4} \mathrm{o}$ valor 0,5 para a razão $\mathrm{MP}_{2,5} /$ $\mathrm{MP}_{10}$ é típico das áreas urbanas dos países em desenvolvimento e é considerado ser o limite inferior da faixa normalmente observada em países desenvolvidos $(0,5-0,8)$. De acordo com a CETESB,${ }^{25}$ na
RMSP, cerca de $60 \%$ da massa do $\mathrm{MP}_{10}$ é constituída de $\mathrm{MP}_{2,5}$. Em Niterói (RJ), essa proporção é menor (em torno de 40\%), provavelmente, devido à proximidade do mar e, consequentemente, maior influência das partículas geradas pelos borrifos de água do mar, que predominam na fração grossa do $\mathrm{MP}_{10}{ }^{8}{ }^{8}$

\section{Fração inorgânica solúvel em água do MP}

As medidas de acidez livre dos extratos aquosos revelaram que o $\mathrm{pH}$ médio ( \pm desvio padrão) para o $\mathrm{MP}_{2,5}$ foi 5,45 \pm 0,5 (mediana $=5,68$ ), variando de 4,63 a 7,18, correspondendo a uma concentração de $\mathrm{H}^{+}$de $3,58 \mu \mathrm{mol} \mathrm{L}{ }^{-1}$. O pH médio (média aritmética) foi calculado a partir das concentrações de $\mathrm{H}^{+}$. No caso do $\mathrm{MP}_{2,5-10}$, o $\mathrm{pH}$ médio foi 5,58 \pm 0,5 (mediana $=5,80$ ), variando de 4,73 a 7,10, correspondendo a uma concentração de $\mathrm{H}^{+}$de $2,66 \mu \mathrm{mol} \mathrm{L} \mathrm{L}^{-1}$. Portanto, $\mathrm{MP}_{2,5}$ e $\mathrm{MP}_{2,5-10}$ apresentaram graus de acidez de seus extratos aquosos muito semelhantes. As condutividades médias foram 3,8 $\pm 1,8 \mu \mathrm{S} \mathrm{cm}^{-1}$ para o $\mathrm{MP}_{2,5}$ e 2,5 \pm 0,9 $\mu \mathrm{S} \mathrm{cm}^{-1}$ para o $\mathrm{MP}_{2,5-10}$, o que indica que, comparativamente, o $\mathrm{MP}_{2,5}$ apresenta uma quantidade de íons solúveis em água superior a do $\mathrm{MP}_{2,5-10}{ }^{\circ}$

Na Tabela 2 encontram-se compiladas as concentrações $\left(\mathrm{em} \mu \mathrm{g} \mathrm{m}^{-3}\right)$ dos íons inorgânicos solúveis em água associados ao $\mathrm{MP}_{2,5} \mathrm{e} \mathrm{MP}_{2,5-10}$. Nessa tabela não se incorporaram as concentrações de $\mathrm{H}^{+}$, cujas médias aritméticas das frações $\mathrm{MP}_{2,5}\left(0,004 \mu \mathrm{g} \mathrm{m}^{-3}\right)$ e $\mathrm{MP}_{2,5-10}\left(0,003 \mu \mathrm{g} \mathrm{m}^{-3}\right)$, quando expressas em unidade mássica, são muito pequenas quando comparadas às dos demais íons. As distribuições desses íons na fração solúvel, com base nas concentrações molares, são mostradas nas Figuras 1A e 1B. Em unidade equivalente, a diferença entre o somatório de cátions (inclusive $\mathrm{H}^{+}$) e ânions associados ao $\mathrm{MP}_{2,5}$ resulta em um

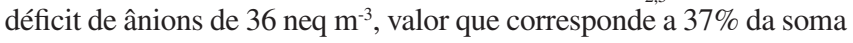
dos cátions. No caso do $\mathrm{MP}_{2,5-10}$, ocorre também um déficit de ânions, de 34 neq $\mathrm{m}^{-3}$, correspondendo a $50 \%$ da soma dos cátions. Tais déficits de ânions podem ser atribuídos à presença de íons não determinados, tais como, (bi)carbonatos e carboxilatos. ${ }^{11}$
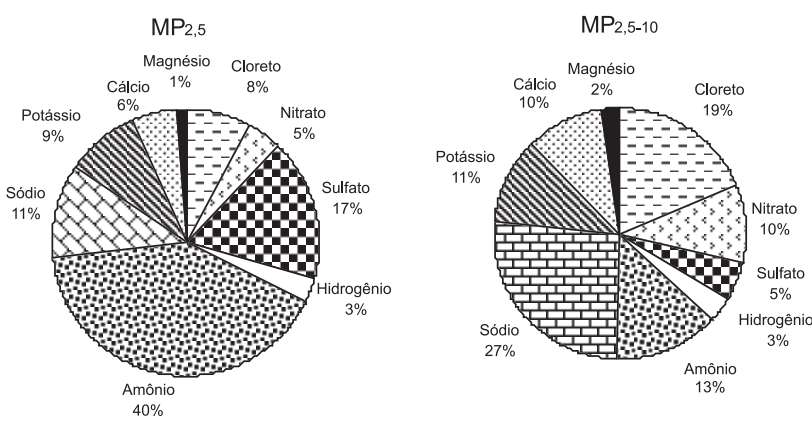

Figura 1. Distribuição percentual das concentrações dos principais constituintes inorgânicos solúveis em água associados ao $M P$ fino $\left(M P_{2,5}\right)$ e grosso $\left(M_{2,5-10}\right)$. Os valores foram calculados com base nas concentrações molares

Os íons $\mathrm{Cl}^{-}, \mathrm{SO}_{4}^{2-}, \mathrm{Na}^{+}, \mathrm{K}^{+}, \mathrm{Ca}^{2+}$ e $\mathrm{Mg}^{2+}$ presentes no $\mathrm{MP}$ atmosférico podem ter como origem a água do mar. Os elementos $\mathrm{Na}, \mathrm{K}$, Mg e Ca podem também constituir a fração insolúvel em água do MP atmosférico quando associados à estrutura de minerais formadores da crosta terrestre, como a dos feldspatos, por exemplo. $\mathrm{O}$ íon $\mathrm{Na}^{+}$ é frequentemente utilizado como traçador conservativo da água do mar em amostras de água de chuva e MP atmosférico, ${ }^{8,22,23,27,28}$ sendo assim, a parcela de origem marinha dos íons $\mathrm{Cl}^{-}, \mathrm{SO}_{4}^{2-}, \mathrm{K}^{+}, \mathrm{Ca}^{2+} \mathrm{e}$ $\mathrm{Mg}^{2+}$ pode ser calculada através da Equação $1: 28$

$$
[\mathrm{X}]_{\text {mar }}=\left\{[\mathrm{X}] /\left[\mathrm{Na}^{+}\right]\right\}_{\text {mar }} \times\left[\mathrm{Na}^{+}\right]_{\text {analisado }}
$$

na qual $[\mathrm{X}]_{\operatorname{mar}}$ representa a concentração de um dos íons - $\mathrm{Cl}^{-}, \mathrm{Mg}^{2+}$, 
Tabela 2. Concentrações $\left(\mu \mathrm{g} \mathrm{m}^{-3}\right)$ dos íons inorgânicos solúveis em água associados ao $\mathrm{MP}$ fino $\left(\mathrm{MP}_{2,5}\right)$ e grosso $\left(\mathrm{MP}_{2,5-10}\right)$ em São José dos Campos

\begin{tabular}{lcccccccc}
\hline & $\mathrm{Cl}^{-}$ & $\mathrm{NO}_{3}^{-}$ & $\mathrm{SO}_{4}^{2-}$ & $\mathrm{NH}_{4}^{+}$ & $\mathrm{Na}^{+}$ & $\mathrm{K}^{+}$ & $\mathrm{Ca}^{2+}$ & $\mathrm{Mg}^{2+}$ \\
\hline $\mathrm{MP}_{2,5}$ & & & & & & & \\
Média \pm DP & $0,36 \pm 0,28$ & $0,37 \pm 0,27$ & $2,17 \pm 1,37$ & $0,96 \pm 0,59$ & $0,34 \pm 0,26$ & $0,46 \pm 0,28$ & $0,30 \pm 0,15$ & $0,03 \pm 0,01$ \\
Mediana & 0,28 & 0,32 & 1,75 & 0,95 & 0,27 & 0,41 & 0,28 \\
Mínimo & $<0,24$ & $<0,02$ & $<0,06$ & $<0,02$ & $<0,17$ & $<0,11$ & $<0,09$ & 0,03 \\
Máximo & 1,40 & 1,03 & 5,41 & 3,52 & 1,08 & 1,26 & 0,62 & \\
& & & & & & & \\
$\mathrm{MP}_{2,5-10}$ & & & & & & & \\
Média \pm DP & $0,5 \pm 0,51$ & $0,54 \pm 0,35$ & $0,44 \pm 0,30$ & $0,21 \pm 0,17$ & $0,56 \pm 0,45$ & $0,37 \pm 0,18$ & $0,38 \pm 0,18$ & $0,05 \pm 0,03$ \\
Mediana & 0,41 & 0,52 & 0,37 & 0,17 & 0,42 & 0,30 & 0,35 & 0,04 \\
Mínimo & $<0,24$ & $<0,02$ & $<0,06$ & $<0,02$ & $<0,17$ & $<0,11$ & $<0,09$ & 0,02 \\
Máximo & 2,35 & 1,58 & 1,26 & 0,70 & 2,39 & 0,96 & 0,89 & 0,16 \\
\hline
\end{tabular}

DP: desvio padrão.

$\mathrm{Ca}^{2+}, \mathrm{K}^{+}$ou $\mathrm{SO}_{4}^{2-}$ - presentes no MP cuja origem é atribuída exclusivamente à água do mar, $\left\{[\mathrm{X}] /\left[\mathrm{Na}^{+}\right]\right\}_{\operatorname{mar}}$ é a razão entre as concentrações dos íons $\mathrm{X} \mathrm{e} \mathrm{Na}^{+}$na água do mar e $\left[\mathrm{Na}^{+}\right]_{\text {analisado }}$ corresponde à concentração do íon $\mathrm{Na}^{+}$determinada no MP. Vale ressaltar que os valores de $\left\{[\mathrm{X}] /\left[\mathrm{Na}^{+}\right]\right\}_{\text {mar }}$ podem variar em função da unidade utilizada para expressar as concentrações dos íons em questão. Portanto, para [X] ${ }_{\text {mar }} \mathrm{e}\left[\mathrm{Na}^{+}\right]_{\text {analisado }}$ expressos em unidade molar (e.g., $\mathrm{nmol} \mathrm{m}^{-3}$ ou $\mu \mathrm{mol}$ $\mathrm{m}^{-3}$ ), as razões molares $\left\{[\mathrm{X}] /\left[\mathrm{Na}^{+}\right]\right\}_{\text {mar }}$ para os íons $\mathrm{Cl}^{-}, \mathrm{Mg}^{2+}, \mathrm{Ca}^{2+}, \mathrm{K}^{+}$ e $\mathrm{SO}_{4}{ }^{2-}$ são, respectivamente, 1,$1637 ; 0,1126 ; 0,0219 ; 0,0218$ e 0,0602 , calculados com base nas concentrações desses íons na água do mar. ${ }^{29,30}$

A diferença entre a concentração do íon X determinado no MP, designada por $[\mathrm{X}]_{\text {total }}$, e a concentração atribuída à água do mar, [X] mar (Equação 1) fornece a concentração do íon $\mathrm{X}$ atribuída a outras fontes (naturais e/ou antrópicas). Essa diferença é designada concentração em excesso (em relação à água do mar), representada por $[\mathrm{X}]_{\text {excesso }}$ na Equação 2:

$$
[\mathrm{X}]_{\text {excesso }}=[\mathrm{X}]_{\text {total }}-[\mathrm{X}]_{\mathrm{mar}}
$$

As distribuições das concentrações dos íons $\mathrm{Cl}^{-}, \mathrm{SO}_{4}^{2-}, \mathrm{K}^{+}, \mathrm{Ca}^{2+}$ e $\mathrm{Mg}^{2+}$ no $\mathrm{MP}_{2,5} \mathrm{e} \mathrm{MP}_{2,5-10}$ atribuídas à água do mar e a outras fontes (naturais e/ou antrópicas) estão representadas nas Figuras 2A e 2B. Doravante, a parcela do íon X cuja origem não está associada à água do mar será abreviada como exc-X.

Nas Figuras 2A e 2B observa-se que a fração em excesso é a predominante para os íons $\mathrm{SO}_{4}^{2-}, \mathrm{K}^{+} \mathrm{e} \mathrm{Ca}^{2+}$ nas duas faixas de tamanho de MP. Os íons $\mathrm{NO}_{3}{ }^{-}$e $\mathrm{NH}_{4}{ }^{+}$encontram-se presentes na água do mar em concentrações traços e muito variáveis $\left(<1-500 \mu \mathrm{g} \mathrm{N} \mathrm{L}^{-1}\right),{ }^{29,30}$ portanto, considera-se que suas origens não têm relação direta com o aerossol de sal marinho. Sendo assim, possivelmente decorrem das emissões dos gases $\mathrm{NO}_{x}$ e amônia $\left(\mathrm{NH}_{3}\right)$. O déficit de $\mathrm{Cl}^{-}$, observado nas duas faixas de tamanho, resulta possivelmente de reações químicas na atmosfera. Especialmente na atmosfera poluída, essa perda pode ser atribuída à volatilização do ácido clorídrico $(\mathrm{HCl})$ resultante das reações entre o cloreto de sódio $(\mathrm{NaCl})$, oriundo da água do mar, e os ácidos nítrico $\left(\mathrm{HNO}_{3}\right)$ e sulfúrico $\left(\mathrm{H}_{2} \mathrm{SO}_{4}\right): 1,31,32$

$\mathrm{NaCl}(\mathrm{s})+\mathrm{HNO}_{3}(\mathrm{~g}) \rightarrow \mathrm{NaNO}_{3}(\mathrm{~s})+\mathrm{HCl}(\mathrm{g})$

$2 \mathrm{NaCl}(\mathrm{aq})+\mathrm{H}_{2} \mathrm{SO}_{4}(\mathrm{aq}) \rightarrow(\mathrm{Na})_{2} \mathrm{SO}_{4}(\mathrm{aq})+2 \mathrm{HCl}(\mathrm{g})$

As cinéticas e os mecanismos dessas e de outras reações, bem como de seus principais fatores de controle, que podem conduzir à formação e volatilização de $\mathrm{HCl}$ (e de outras formas voláteis de cloro), são amplamente discutidos nos trabalhos de FinlaysonPitts $^{31}$ e Rossi. ${ }^{32} \mathrm{O}$ pequeno déficit observado para o $\mathrm{Mg}^{2+}$ no MP
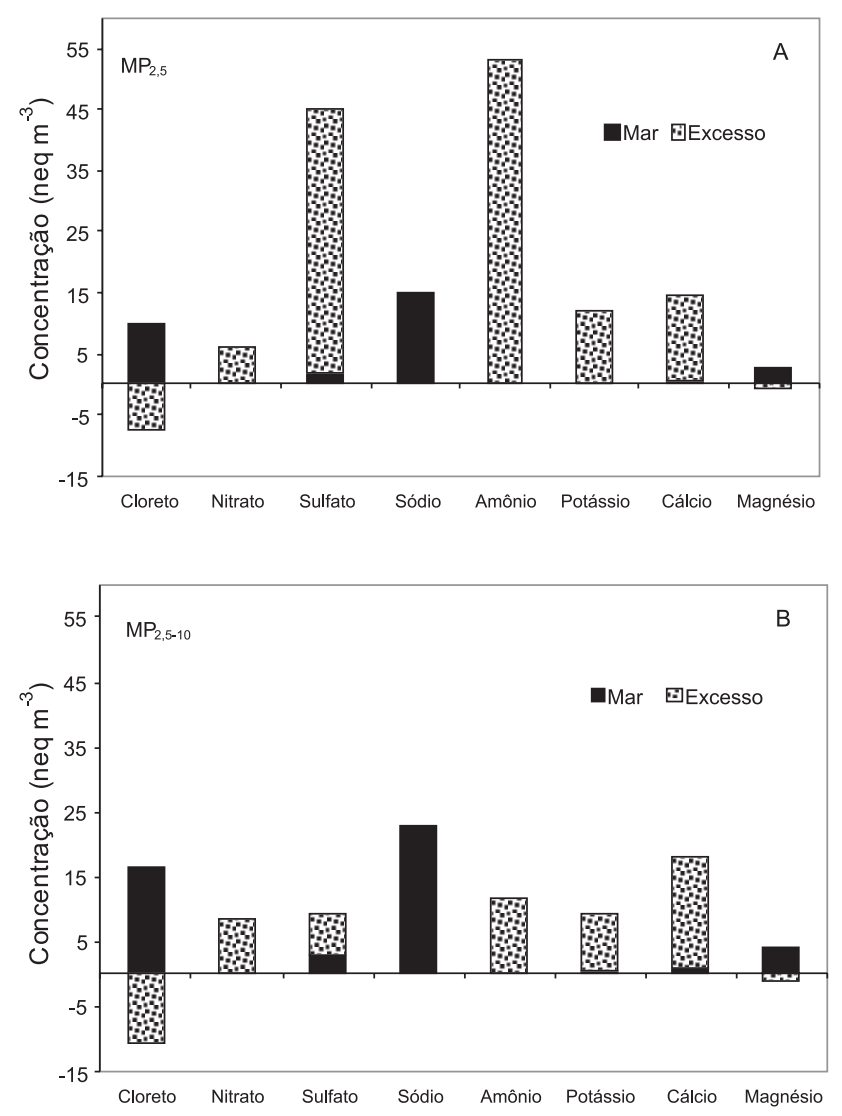

Figura 2. Concentrações médias dos íons inorgânicos e representação das parcelas oriundas dos aerossóis de sal marinho (Mar) e aos excessos (Excesso) associados ao MP fino (A) e grosso (B). Valores negativos representam déficit em relação à parcela total originária do sal marinho

encontra-se dentro da faixa de incerteza dos resultados face à sua baixa concentração média.

\section{Material particulado fino}

A massa do total dos íons inorgânicos solúveis em água corresponde a $32 \%$ da massa total do $\mathrm{MP}_{2,5}$. Em unidade molar, as concentrações médias dos íons no $\mathrm{MP}_{2,5}$ decresceram na seguinte ordem $\mathrm{NH}_{4}^{+}>\mathrm{SO}_{4}{ }^{2-}>\mathrm{Na}^{+}>\mathrm{K}^{+}>\mathrm{Cl}^{-}>\mathrm{Ca}^{2+}>\mathrm{NO}_{3}^{-}>\mathrm{H}^{+}>\mathrm{Mg}^{2+}$ (Figura 1A).

Os íons $\mathrm{NH}_{4}^{+} \mathrm{e} \mathrm{SO}_{4}^{2-}$ compreende juntos $20 \%$ da massa total do $\mathrm{MP}_{2,5}$ e $57 \%$ da massa total da fração inorgânica solúvel associada a essa faixa de tamanho. No que diz respeito a estudos realizados 
no Brasil, a predominância desses íons no $\mathrm{MP}_{2,5}$ foi igualmente encontrada por Bourotte et al., ${ }^{7}$ na cidade de São Paulo, e Mariani e de Mello, ${ }^{8}$ em Niterói (Tabela 3 ). A concentração média de $\mathrm{SO}_{4}{ }^{2-}$ no $\mathrm{MP}_{2,5}$ em SJC foi maior do que a verificada na cidade de São Paulo ${ }^{7}$ e menor que a de Niterói. ${ }^{8}$ A concentração de $\mathrm{NH}_{4}^{+}$em SJC foi três vezes maior que a verificada na cidade de São Paulo ${ }^{7}$ e muito semelhante à de Niterói ${ }^{8}$ (Tabela 3 ).

Tabela 3. Comparação das concentrações $\left(\mu \mathrm{g} \mathrm{m}^{-3}\right)$ dos íons inorgânicos majoritários associados ao $\mathrm{MP}_{2,5}$ e $\mathrm{MP}_{2,5-10}$ entre São José dos Campos e outras localidades no Brasil

\begin{tabular}{lcccccccccc}
\hline & $\mathrm{Cl}^{-}$ & $\mathrm{NO}_{3}^{-}$ & $\mathrm{SO}_{4}^{2-}$ & $\mathrm{NH}_{4}^{+}$ & $\mathrm{Na}^{+}$ & $\mathrm{K}^{+}$ & $\mathrm{Ca}^{2+}$ & $\mathrm{Mg}^{2+}$ & Ref. \\
\hline $\begin{array}{l}\mathrm{MP}_{2,5} \\
\begin{array}{l}\text { São José dos } \\
\text { Campos }\end{array}\end{array}$ & 0,36 & 0,37 & 2,17 & 0,96 & 0,34 & 0,46 & 0,30 & 0,03 & $\begin{array}{c}\text { Este } \\
\text { trabalho }\end{array}$ \\
$\begin{array}{l}\text { São Paulo } \\
\text { Niterói }\end{array}$ & 0,12 & 0,43 & 1,20 & 0,30 & 0,13 & 0,14 & 0,10 & 0,02 & $\begin{array}{c}\text { Bourotte } \\
\text { et al. }\end{array}$ \\
& 0,29 & 0,40 & 2,56 & 0,94 & 0,33 & 0,24 & 0,05 & 0,05 & $\begin{array}{l}\text { Mariani } \\
\text { ede Mello }\end{array}$
\end{tabular}

$\mathrm{MP}_{2,5-10}$

São José dos $\quad 0,58 \quad 0,54 \quad 0,44 \quad 0,21 \quad 0,56 \quad 0,37 \quad 0,38 \quad 0,05 \quad$ Este

Campos

trabalho

São Paulo

$\begin{array}{llllllll}0,29 & 1,04 & 0,87 & 0,02 & 0,11 & 0,08 & 0,39 & 0,08\end{array}$

Bourotte

et al. ${ }^{7}$

Niterói

$\begin{array}{lllllllll}2,96 & 1,39 & 1,12 & 0,32 & 1,83 & 0,25 & 0,26 & 0,49 & \text { Mariani }\end{array}$ e de Mello ${ }^{8}$

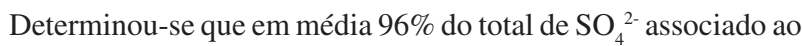
$\mathrm{MP}_{2,5}$ representam exc- $\mathrm{SO}_{4}{ }_{4}^{2-}$, significando que a maior parte do $\mathrm{SO}_{4}{ }^{2-}$ nessa faixa de tamanho originou-se da oxidação do $\mathrm{SO}_{2}$. Na atmosfera, a $\mathrm{NH}_{3}$ reage preferencialmente com o $\mathrm{H}_{2} \mathrm{SO}_{4}$ (formado a partir da oxidação do $\mathrm{SO}_{2}$ ), dando origem progressivamente, dependendo da abundância de $\mathrm{NH}_{3}$ no ar, aos sais bissulfato de amônio $\left(\mathrm{NH}_{4} \mathrm{HSO}_{4}\right)$ e sulfato de amônio $\left(\left(\mathrm{NH}_{4}\right)_{2} \mathrm{SO}_{4}\right){ }^{2}$ Havendo excesso de $\mathrm{NH}_{3}$ no ar após todo $\mathrm{H}_{2} \mathrm{SO}_{4}$ já ter sido exaurido, esta reagirá em fase gasosa com o $\mathrm{HNO}_{3}$ (formado a partir da oxidação do $\mathrm{NO}_{x}$ ), formando nitrato de amônio $\left(\mathrm{NH}_{4} \mathrm{NO}_{3}\right)$. Entretanto, a estabilidade do $\mathrm{NH}_{4} \mathrm{NO}_{3}$ reduz com o aumento da temperatura e diminuição da umidade relativa do ar, condições que favorecem a sua decomposição às formas gasosas $\mathrm{NH}_{3}$ e $\mathrm{HNO}_{3}{ }^{2}$ Esses sais de enxofre e nitrogênio formam partículas secundárias, assim chamadas por se formarem na atmosfera através do processo de conversão de gases em partículas (gas-to-particle conversion), que compõem a faixa de tamanho das partículas finas.

De certa forma, o balanço de carga entre alguns íons é uma ferramenta útil para ser inferida na composição das substâncias solúveis que constituem o material particulado. A Figura 3A mostra uma relação linear estatisticamente significativa entre as concentrações de $\mathrm{NH}_{4}^{+}$e de exc- $\mathrm{SO}_{4}{ }^{2-}(\mathrm{r}=0,65 ; \mathrm{P}<0,01)$. Em média, a razão equivalente $\mathrm{NH}_{4}^{+}$/exc- $\mathrm{SO}_{4}{ }^{2-}$ encontrada é 1,23 (razão molar = 2,45), o que sugere que no $\mathrm{MP}_{2,5}$ a maior parte do $\mathrm{NH}_{4}^{+}$deva estar sob a forma de $\left(\mathrm{NH}_{4}\right)_{2} \mathrm{SO}_{4}$, havendo ainda um excesso de $\mathrm{NH}_{4}^{+}$. A Figura 3B mostra a relação entre o $\mathrm{NH}_{4}^{+}$e o exc- $\mathrm{SO}_{4}{ }^{2-}$ e $\mathrm{NO}_{3}^{-}$juntos $(\mathrm{r}=0,62 ; \mathrm{P}<0,01)$. Nesse caso, a razão equivalente $\mathrm{NH}_{4}^{+} /($exc$\mathrm{SO}_{4}{ }^{2-}+\mathrm{NO}_{3}^{-}$) é 1,08 (razão molar $=1,92$ ), sugerindo a existência de partículas finas de $\left(\mathrm{NH}_{4}\right)_{2} \mathrm{SO}_{4}$ e $\mathrm{NH}_{4} \mathrm{NO}_{3}$. A pequena parcela ainda excedente de $\mathrm{NH}_{4}^{+}$em relação ao $\mathrm{NO}_{3}^{-}$e exc- $\mathrm{SO}_{4}{ }^{2-}$ juntos pode ser explicada como resultado de erros associados às determinações desses íons e/ou pela presença no $\mathrm{MP}_{2,5}$ de outros sais formados por ânions não analisados, como carboxilatos de amônio. Em valor absoluto, o excedente de $\mathrm{NH}_{4}{ }^{+}$relativo à soma $\left(\right.$exc- $\left.\mathrm{SO}_{4}{ }^{2-}+\mathrm{NO}_{3}{ }^{-}\right)$ representa em média 4 neq $\mathrm{m}^{-3}$.
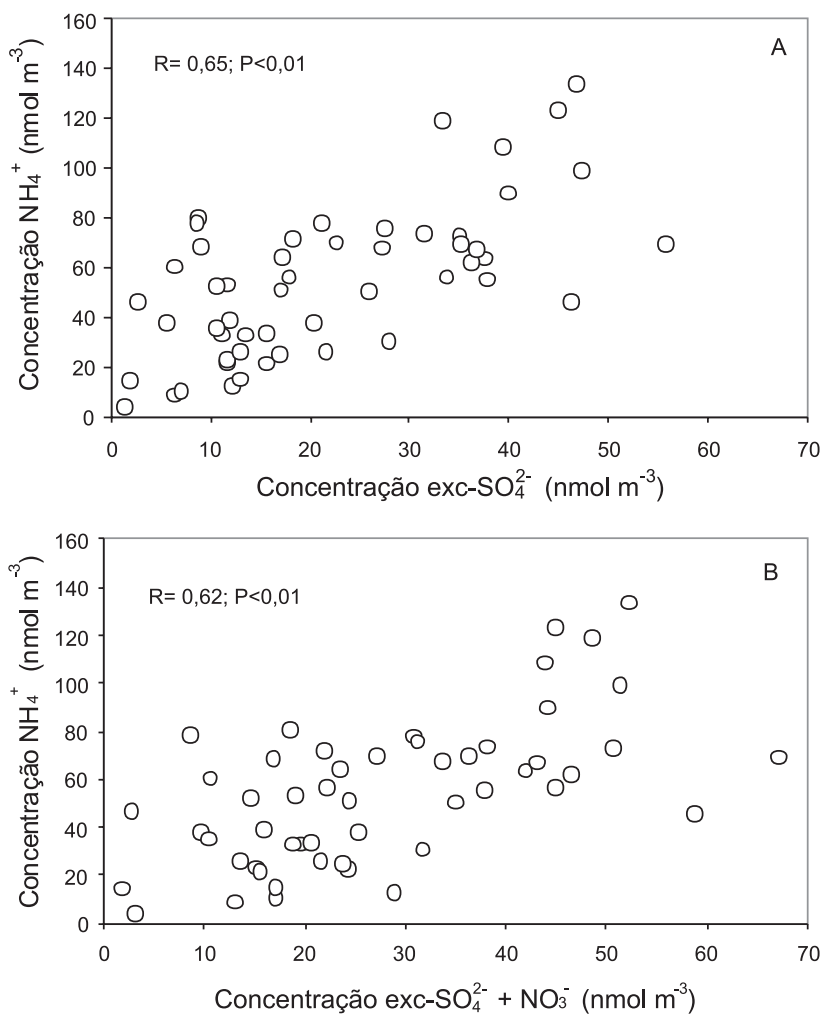

Figura 3. Relação entre as concentrações de amônio e excesso de sulfato (A), e amônio e a soma do excesso de sulfato e nitrato (B), associados ao MP fino

Se em SJC o $\mathrm{NH}_{4}^{+}$associado ao $\mathrm{MP}_{2,5}$ estiver predominantemente sob a forma de $\left(\mathrm{NH}_{4}\right)_{2} \mathrm{SO}_{4}$, estima-se, com base na concentração molar de exc- $\mathrm{SO}_{4}{ }^{2-}$, que a concentração desse sal seja em média 2,9 $\mu \mathrm{g} \mathrm{m}^{-3}$. Esse valor é ligeiramente inferior ao estimado por Mariani e de Mello ${ }^{8}$ em Niterói, que foi $3,4 \mu \mathrm{g} \mathrm{m}^{-3}$. A concentração média de $\left(\mathrm{NH}_{4}\right)_{2} \mathrm{SO}_{4}$ no $\mathrm{MP}_{2,5}$ em SJC é superior às observadas em ambientes tropicais ainda pouco impactados pelas atividades humanas e intermediária em relação à faixa verificada em países desenvolvidos. Por exemplo, medidas de $\mathrm{MP}_{2,5}$ realizadas por Graham et al. ${ }^{33}$ nas proximidades de Balbina ( $100 \mathrm{~km}$ ao norte de Manaus), em julho de 2001, mostraram concentrações médias de $\left(\mathrm{NH}_{4}\right)_{2} \mathrm{SO}_{4}$ de $0,5 \mu \mathrm{g}$ $\mathrm{m}^{-3}\left(15 \%\right.$ da massa do $\left.\mathrm{MP}_{2,5}\right)$ durante o dia e $0,3 \mu \mathrm{g} \mathrm{m}^{-3}$ durante a noite $\left(13 \%\right.$ da massa do $\left.\mathrm{MP}_{2,5}\right)$. Em amostras de $\mathrm{MP}_{2,5}$ coletadas (em 2001) em 143 pontos distribuídos pelos Estados Unidos, Malm et al..$^{34}$ encontraram concentrações médias anuais de $\left(\mathrm{NH}_{4}\right)_{2} \mathrm{SO}_{4}$ que variaram de $0,3 \mu \mathrm{g} \mathrm{m}^{-3}$ (Tapper Creek, Alaska) até $6,4 \mu \mathrm{g} \mathrm{m}^{-3}$ (Ohio River Valley, Indiana). Na parte central e oeste dos Estados Unidos, as concentrações foram em geral inferiores a $1 \mu \mathrm{g} \mathrm{m}^{-3}$, enquanto que no centro-leste estiveram em torno de $5 \mu \mathrm{g} \mathrm{m}^{-3}$.

A concentração média de $\mathrm{Na}^{+}$no $\mathrm{MP}_{2,5}$ em SJC é muito semelhante àquela encontrada em Niterói ${ }^{8}$ e aproximadamente 3 vezes maior que a da cidade de São Paulo. ${ }^{7} \mathrm{O}$ mesmo se verifica para os teores de $\mathrm{Cl}^{-}$, embora os valores obtidos em SJC sejam ligeiramente superiores quando comparados aos de Niterói. Isso pode estar associado à diferença no regime de distribuição sazonal das chuvas entre SJC e Niterói, uma vez que a precipitação contribui para remoção de partículas da atmosfera. De acordo com as séries históricas de precipitação, em média, a quantidade anual de chuva precipitada em SJC $(1.282 \mathrm{~mm}){ }^{35}$ é ligeiramente acima daquela registrada em Niterói $(1.225 \mathrm{~mm}),{ }^{36}$ entretanto, no período de maio a setembro (período de estiagem) as séries históricas mostram que em Niterói a precipitação acumulada é de $292 \mathrm{~mm},{ }^{36}$ enquanto que em SJC, no mesmo período, é de $236 \mathrm{~mm} .{ }^{35}$ A maior precipitação pode levar a 
menores concentrações atmosféricas de $\mathrm{Na}^{+}$e $\mathrm{Cl}^{-}$, devido à ação de limpeza da atmosfera pela chuva. O déficit médio de $\mathrm{Cl}^{-}$no $\mathrm{MP}_{2,5} \mathrm{em}$ SJC (41\%) foi inferior ao observado em Niterói (51\%). ${ }^{8} \mathrm{O}$ processo representado pela Equação 3 deve justificar esse déficit sendo, inclusive, corroborado pelo fato da razão média $\left(\mathrm{Cl}^{-}+\mathrm{NO}_{3}^{-}\right) / \mathrm{Na}^{+}(=1,09)$ ser próxima à unidade, sugerindo assim que no $\mathrm{MP}_{2,5} \mathrm{o} \mathrm{Na}^{+}$deva estar predominantemente sob a forma de $\mathrm{NaCl}$ e $\mathrm{NaNO}_{3}$.

As concentrações de $\mathrm{K}^{+} \mathrm{e} \mathrm{Ca}^{2+}$ associadas ao $\mathrm{MP}_{2,5}$ em SJC foram superiores àquelas verificadas nas cidades de Niterói ${ }^{8}$ e São Paulo, ${ }^{7}$ possivelmente, devido ao transporte de partículas finas oriundas da queima de material vegetal. A concentração média de $\mathrm{K}^{+}$no $\mathrm{MP}_{2,5}$ é 1,5 vezes superior a do $\mathrm{Ca}^{2+}$ e a parcela atribuída ao exc- $\mathrm{Ca}^{2+} \mathrm{e}$ exc- $\mathrm{K}^{+}$ associado à essa faixa de tamanho é de 96-97\%. Em Piracicaba ( 220 $\mathrm{km}$ a oeste-noroeste de SJC), Lara et al. ${ }^{37}$ encontraram concentrações médias anuais de $\mathrm{K}$ e $\mathrm{Ca}$ de 0,36 e $0,08 \mu \mathrm{g} \mathrm{m}^{-3}$, respectivamente, associadas ao $\mathrm{MP}_{2.5}$, cuja média anual foi $16,1 \mu \mathrm{g} \mathrm{m}^{-3}$, cuja origem foi atribuída predominantemente à queima da cana-de-açúcar.

\section{Material particulado grosso}

A massa do total dos íons inorgânicos solúveis em água corresponde a $21 \%$ da massa total do $\mathrm{MP}_{2,5-10}$. Em unidade molar, as concentrações médias dos íons decresceram na seguinte ordem $\mathrm{Na}^{+}$ $>\mathrm{Cl}^{-}>\mathrm{NH}_{4}^{+}>\mathrm{K}^{+}>\mathrm{Ca}^{2+} \sim \mathrm{NO}_{3}{ }^{-}>\mathrm{SO}_{4}{ }^{2-}>\mathrm{H}^{+}>\mathrm{Mg}^{2+}$. A Figura $1 \mathrm{~B}$ mostra suas distribuições e os correspondentes valores relativos.

Os íons $\mathrm{Na}^{+}$e $\mathrm{Cl}^{-}$foram os mais abundantes na fração grossa, formando juntos $46 \%$ da massa total de íons inorgânicos solúveis analisados e 7,5\% da massa do $\mathrm{MP}_{2,5-10}$, sugerindo uma significativa influência dos aerossóis de sal marinho nessa faixa de tamanho do MP. As concentrações médias desses dois íons encontram-se numa faixa intermediária entre aquelas verificadas na cidade de São Paulo ${ }^{7}$ e em Niterói ${ }^{8}$ (Tabela 3 ).

No $\mathrm{MP}_{25-10}$ o déficit médio de $\mathrm{Cl}^{-}$foi de $39 \%$, idêntico àquele verificado no $\mathrm{MP}_{2,5}$. A concentração média de $\mathrm{NO}_{3}{ }^{-}$no $\mathrm{MP}_{2,5-10}(0,54$ $\left.\mu \mathrm{g} \mathrm{m}^{-3}\right)$ é, entretanto, mais alta do que no $\mathrm{MP}_{2,5}\left(0,37 \mu \mathrm{g} \mathrm{m}^{-3}\right)$. No $\mathrm{MP}_{2,5-10}$, a razão molar $\left(\mathrm{Cl}^{-}+\mathrm{NO}_{3}^{-}\right) / \mathrm{Na}^{+}(=1,03)$ se aproxima da unidade e, portanto, corrobora o processo representado pela Equação 3 como o principal responsável pelo déficit de $\mathrm{Cl}^{-}$no $\mathrm{MP}$ grosso. Esse processo contribui para a remoção de parte do $\mathrm{HNO}_{3}$ gasoso da atmosfera, decorrente da oxidação dos óxidos de nitrogênio emitidos pelos processos de combustão. Sob a forma de partículas de $\mathrm{NaNO}_{3}$, o $\mathrm{NO}_{3}$ - é removido da atmosfera por deposição seca e úmida.

A concentração média de $\mathrm{Ca}^{2+}$ no $\mathrm{MP}_{2,5-10}$ em SJC foi muito semelhante àquela verificada na cidade de $\mathrm{São} \mathrm{Paulo}^{7}$, mas superior à de Niterói ${ }^{8}$ (Tabela 3 ). Os teores de $\mathrm{K}^{+}$foram maiores em SJC comparados a essas duas localidades, ${ }^{7,8}$ com uma diferença mais acentuada em relação à São Paulo (Tabela 3). Em estudo realizado na Mata Atlântica, Artaxo e Orsini ${ }^{38}$ sugerem que a vegetação seja uma importante fonte de partículas de K e Ca para a atmosfera. Durante a transpiração sais solúveis de $\mathrm{K}$ e Ca tendem a se acumular sobre a superfície das folhas e, por conseguinte, podem se dispersar para a atmosfera pela ação dos ventos. ${ }^{39}$ Uma forte evidência desse acúmulo é o enriquecimento em $\mathrm{K}$ e Ca que se verifica na água da chuva após a passagem pela copa da floresta. ${ }^{40}$ Concentrações médias anuais mais elevadas de $\mathrm{Ca}\left(0,67 \mu \mathrm{g} \mathrm{m}^{-3}\right)$ e $\mathrm{K}\left(0,46 \mu \mathrm{g} \mathrm{m}^{-3}\right)$ no $\mathrm{MP}_{2,5-10}$ que as encontradas em SJC foram relatadas por Lara et al. ${ }^{37} \mathrm{em}$ Piracicaba, cujas origens foram atribuídas ao solo e à queima de cana-de-açúcar, respectivamente. Outras possíveis fontes antrópicas de $\mathrm{Ca}^{2+} \mathrm{e} \mathrm{K}^{+}$nos grandes centros urbanos são o uso do cimento na construção civil e a incineração de lixo urbano.

\section{CONCLUSÕES}

Concentrações de MP inalável $\left(\mathrm{MP}_{10}\right)$, subdivididos como fino
$\left(\mathrm{MP}_{2,5}\right)$ e grosso $\left(\mathrm{MP}_{2,5-10}\right)$, foram medidas em São José dos Campos (SJC) no período de fevereiro de 2004 a fevereiro de 2005. As concentrações médiasde $24 \mathrm{~h}$ de $\mathrm{MP}_{10}$ variaram de 3,0 a 71,9 $\mu \mathrm{g} \mathrm{m}^{-3}$, com média anual de $31,2 \mu \mathrm{g} \mathrm{m}^{-3}$, da qual $52 \%$ foi atribuída ao $\mathrm{MP}_{2,5}$.

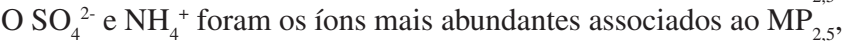
constituindo em média $20 \%$ da massa total do particulado fino e $57 \%$ da massa total dos íons inorgânicos solúveis em água associados a ele. As concentrações de $\mathrm{SO}_{4}{ }^{2-} \mathrm{e} \mathrm{NH}_{4}^{+}$foram 5 vezes maiores no $\mathrm{MP}_{2,5} \mathrm{em}$ relação ao $\mathrm{MP}_{2,5-10}$. Estimou-se uma concentração média de $\left(\mathrm{NH}_{4}\right)_{2} \mathrm{SO}_{4}$ de $2,9 \mu \mathrm{g} \mathrm{m}^{-3}$ no $\mathrm{MP}_{2,5}$. Os íons $\mathrm{Na}^{+}, \mathrm{Cl}^{-} \mathrm{e} \mathrm{NO}_{3}^{-}$foram mais abundantes no $\mathrm{MP}_{2,5-10}$, estando os primeiros possivelmente relacionados ao transporte de aerossóis de sais marinhos e o último a reações entre o $\mathrm{NaCl}$ (s) e o $\mathrm{HNO}_{3}$ (g), resultando na volatilização do $\mathrm{HCl}$. Esse fato é evidenciado pelo déficit de $\mathrm{Cl}^{-}$(em relação à razão $\mathrm{Cl}^{-} / \mathrm{Na}^{+}$na água do mar) encontrado nas duas faixas de tamanho do MP, bem como, pela razão $\left(\mathrm{Cl}^{-}+\mathrm{NO}_{3}^{-}\right) / \mathrm{Na}^{+}$muito próxima à unidade, também verificada nas duas faixas de tamanho do MP. $\mathrm{O} \mathrm{Mg}^{2+}$ foi o íon menos abundante, tanto no $\mathrm{MP}_{2,5}$ quanto no $\mathrm{MP}_{2,5-10}$. As concentrações de $\mathrm{K}^{+}$ e $\mathrm{Ca}^{2+}$ no $\mathrm{MP}_{25}$ e $\mathrm{MP}_{25-10}$ em SJC foram superiores às relatadas por outros estudos realizados em regiões urbanizadas e industrializadas, como a RMSP e a RMRJ, podendo estar relacionadas à uma maior influência de processos biogênicos e atividades antrópicas, como queima de biomassa e processos industriais.

\section{MATERIAL SUPLEMENTAR}

A Figura 1S (Local de amostragem - Instituto Nacional de Pesquisas Espaciais (INPE), São José dos Campos, RJ) está disponível para consulta gratuita em http://quimicanova.sbq.org.br na forma de arquivo PDF.

\section{AGRADECIMENTOS}

Ao Conselho Nacional de Desenvolvimento Científico e Tecnológico $(\mathrm{CNPq})$ pelo apoio financeiro concedido aos projetos 474113/2003-5 e 477073/2007-7.

\section{REFERÊNCIAS}

1. Finlayson-Pitts, B. J.; Pitts Jr., J. N.; Chemistry of the Upper and Lower Atmosphere - Theory, Experiments and Applications, Academic Press: San Diego, 2000.

2. Seinfeld, J. H.; Pandis, S. N.; Atmospheric Chemistry and Physics from Air Pollution to Climate Change, $2^{\text {nd }}$ ed., John Wiley and Sons, Inc.: Hoboken, 2006.

3. http://www.ipcc.ch/pdf/assessment-report/ar4/syr/ar4_syr.pdf, acessada em Outubro 2009.

4. World Health Organization; WHO Air quality guidelines for particulate matter, ozone, nitrogen dioxide and sulfur dioxide, Global update 2005, Summary of risk assessment, WHO Press: Geneva, 2006.

5. http://www.epa.gov/oar/particlepollution/health.html, acessada em Outubro 2009.

6. Castanho, A. D. A.; Artaxo, P.; Atmos. Environ. 2001, 35, 4889; Azevedo, D. A.; Santos, C. Y. M.; Aquino Neto, F. R.; Atmos. Environ. 2002, 36, 2383; Godoy, M. L. D. P.; Godoy, J. M.; Artaxo, P.; Atmos. Environ. 2005, 39, 5307; Miranda, R. M.; Andrade, M. F.; Atmos. Environ. 2005, 39, 6188; Quiterio, S. L.; Sousa, C. R.; Arbilla, G.; Escaleira, V.; Atmos. Environ. 2005, 39, 3503; Pereira, P. A. P.; Lopes, W. A.; Carvalho, L. S.; Rocha, G. O.; Bahia, N. C.; Loyola, J.; Quiterio, S. L.; Escaleira, E.; Arbilla, A.; de Andrade, J. B.; Atmos. Environ. 2007, 41, 7837.

7. Bourotte, C.; Curi-Amarante, A.; Forti, M. C.; Pereira, L. A. A.; Braga, A. L.; Lotuffo, P. A.; Atmos. Environ. 2007, 41, 2036. 
8. Mariani, R. L. N. C.; de Mello, W. Z.; Atmos. Environ. 2007, 41, 2887.

9. Soluri, D. S.; Godoy, M. L. D. P.; Godoy, J. M.; Roldão, L. A.; J. Braz. Chem. Soc. 2007, 18, 838.

10. Paterlini, W. C.; Tese de Doutorado, Universidade Estadual Paulista, Brasil, 2007.

11. Allen, A. G.; Cardoso, A. A.; Rocha, G. O.; Atmos. Environ. 2004, 38, 5025.

12. Yamasoe, M. A.; Artaxo, P.; Miguel, A. H.; Allen, A. G.; Atmos. Environ. 2000, 34, 1641.

13. Souza, I. M.; Dissertação de Mestrado, Universidade do Vale do Paraíba, Brasil, 2003.

14. CETESB - Companhia de Tecnologia e Saneamento Ambiental; Relatório da qualidade do ar no estado de São Paulo 2006, CETESB: São Paulo, 2007.

15. França, D. A.; Dissertação de Mestrado, Instituto Nacional de Pesquisas Espaciais, Brasil, 2007.

16. http://www.sjc.sp.gov.br, acessada em Outubro 2009.

17. http://www.der.sp.gov.br/malha/estat_malha/frotaVeiculos2006.pdf, acessada em Outubro 2009.

18. http://www.epa.gov/ttnamti1/files/ambient/inorganic/mthd-2-2.pdf, acessada em Outubro 2009.

19. Wilson, W. E.; Chow, J. C.; Claiborn, C.; Fusheng, W.; Engelbrecht, J.; Watson, J. G.; Chemosphere 2002, 49, 1009.

20. Eaton, A. D.; Clesceri, L. S.; Greenberg, A. E.; Standard Methods for the Examination of Water and Wastewater, American Public Health Association: Washington, 1995.

21. Grasshoff, K.; Ehrhardt, M.; Kremling, K.; Methods of Sea Water Analysis, $2^{\text {nd }}$ ed., Verlag Chemie: Dearfield Beach, 1983.

22. de Souza, P. A.; de Mello, W. Z.; Maldonado, J.; Evangelista, H.; Quim. Nova 2006, 29, 471.

23. Rodrigues, R. A. R.; de Mello, W. Z.; de Souza, P. A.; Quim. Nova 2007, $30,1842$.

24. http://www.inmetro.gov.br/Sidoq/Arquivos/CGCRE/DOQ/DOQCGCRE-8_02.pdf, acessada em Outubro 2009.
25. CETESB - Companhia de Tecnologia e Saneamento Ambiental; Material particulado inalável fino $\left(M_{2,5}\right)$ e grosso $\left(M P_{2,5-10}\right)$ na atmosfera da Região Metropolitana de São Paulo (2000-2006), CETESB: São Paulo, 2008.

26. http://www.mma.gov.br/port/conama/res/res90/res0390.html, acessada em Outubro 2009.

27. de Mello, W. Z.; Almeida, M. D.; Environ. Pollut. 2004, 129, 63.

28. Keene, W. C.; Pszenny, A. A. P.; Galloway, J. N.; Hawley, M. E.; J. Geophys Res. 1986, 91, 6647.

29. Riley, J. P.; Chester, R.; Introduction to Marine Chemistry, Academic Press: London, 1971.

30. Millero, F. J.; Chemical Oceanography, $3^{\text {rd }}$ ed., Taylor and Francis: Boca Raton, 2006.

31. Finlayson-Pitts, B. J.; Chem. Rev. 2003, 103, 4801.

32. Rossi, M. J.; Chem. Rev. 2003, 103, 4823.

33. Graham, B.; Guyon, P.; Maenhaut, W.; Taylor, P. E.; Ebert, M.; MatthiasMaser, S.; Mayol-Bracero, O. L.; Godoi, R. H. M.; Artaxo, P.; Meixner, F. X.; Moura, M. A. L.; Rocha, C. H. E. D.; van Grieken, R.; Glovsky, M. M.; Flagan, R. C.; Andreae, M. O.; Journey of Geophysical Research (2003), doi:10.1029/2003JD004049.

34. Malm, W. C.; Schichtel, B. A.; Pitchford, M. L.; Ashbaugh, L. L.; Eldred, R. A.; Journey of Geophysical Research (2004), doi:10.1029/2003JD003739.

35. http://www.sigrh.sp.gov.br/cgi-bin/bdhm.exe/plu, acessada em Outubro 2009.

36. FIDERJ - Fundação Instituto de Desenvolvimento Econômico e Social do Estado do Rio de Janeiro; Indicadores Climatológicos, FIDERJ: Rio de Janeiro, 1978.

37. Lara, L. L.; Artaxo, P.; Martinelli, L. A.; Camargo, P. B.; Victoria, R. L.; Ferraz, E. S. B.; Atmos. Environ. 2005, 39, 4627.

38. Artaxo, P.; Orsini, C.; Nuclear Instruments and Methods in Physical Research 1987, 22, 259

39. de Mello, W. Z.; Environ. Poll. 2001, 114, 235.

40. Costa, C. M. P.; de Mello, W. Z.; Geochim. Brasil. 1997, 11, 341. 


\section{CARACTERIZAÇÃO DO MATERIAL PARTICULADO FINO E GROSSO E COMPOSIÇÃO DA FRAÇÃO INORGÂNICA SOLÚVEL EM ÁGUA EM SÃO JOSÉ DOS CAMPOS (SP)}

Patricia Alexandre de Souza e William Zamboni de Mello*

Departamento de Geoquímica, Instituto de Química, Universidade Federal Fluminense, Outeiro de São João Batista, s/n, 24020-141 Niterói - RJ, Brasil

Rauda Lúcia Mariani\#

Centro de Previsão do Tempo e Estudos Climáticos, Instituto Nacional de Pesquisas Espaciais, CP 515, 12227-010 São José dos Campos - SP, Brasil

\section{Silvia Maria Sella}

Departamento de Química Analítica, Instituto de Química, Universidade Federal Fluminense, Outeiro de São João Batista, s/n, 24020-141 Niterói - RJ, Brasil

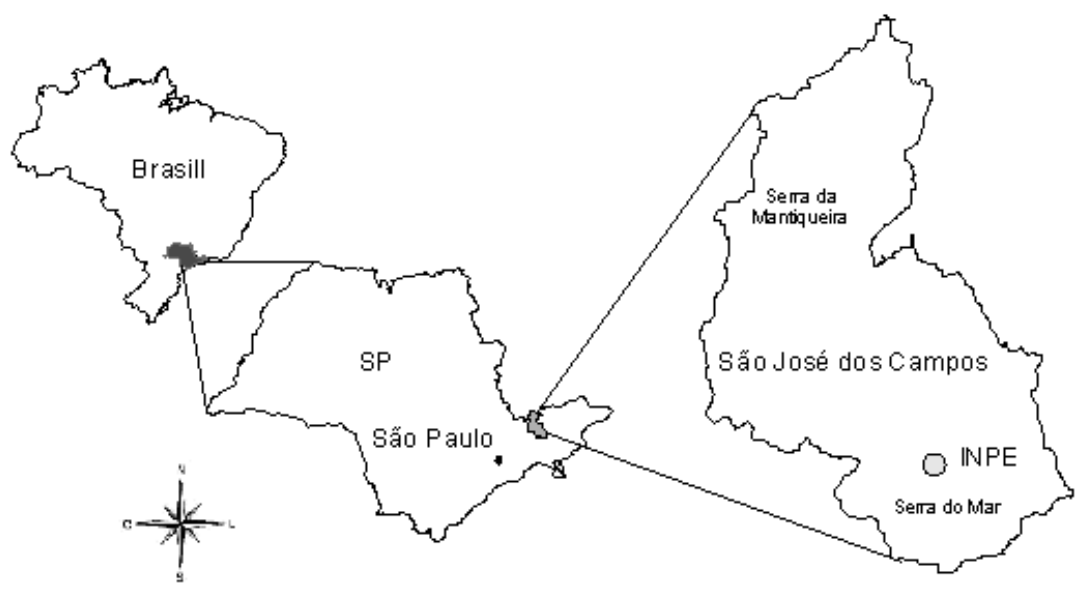

Figura 1S. Local de amostragem - Instituto Nacional de Pesquisas Espaciais (INPE), São José dos Campos, RJ

*e-mail: zamboni@geoq.uff.br

\# Endereço permanente: Departamento de Geoquímica, Instituto de Química, Universidade Federal Fluminense, Outeiro de São João Batista, s/n, 24020141 Niterói - RJ. 